\title{
Radiation Damage in Silicon Trackers at the Tevatron Experiments
}

\author{
Oscar González \\ (On behalf of the CDF and DØ Silicon Groups) \\ CIEMAT, Spain \\ E-mail: oglezefnal.gov
}

\begin{abstract}
An overview of the studies performed on the radiation damage affecting the silicon detectors at the CDF and DØ experiments is presented. These detectors have been exposed to a much larger radiation dose (and operating for a longer time) than originally planned and therefore these studies are fundamental to assess the status and future of the sensors. Results are summarized with special emphasis on the techniques used and their complementarity to achieve a complete picture of the effect of the radiation on the sensors and their performance for physics analysis.
\end{abstract}

VERTEX 2009 (18th workshop)

September 13-18 2009

VELUWE, the Netherlands 


\section{Introduction to the detectors}

The CDF and DØ experiments have been collecting data from the proton-antiproton collisions in Run II of the Tevatron collider (Batavia, Illinois, USA) since 2001. The Tevatron was during decades the most energetic collider in the world providing hadronic collisions at $2 \mathrm{TeV}$ of center-ofmass energy.The high energy and intensity of the beams at this collider represents a great challenge for performing physics with detectors that are close to the beam. On the other hand, an essential part of the Tevatron physics program relies on these detectors to perform measurements requiring very precise tracking close to the interaction point.

For this purpose, both collaborations are making use of silicon-based vertex detectors, located extremely close to the beams and in a harsh environment in terms of the radiation that they have to suffer. Therefore these silicon detectors provide a unique test-bed for the verification of the expectations and understanding of the radiation-hardness in silicon sensors. Much of the information from these studies of the radiation effect on these detectors should be directly applicable for the sensors within the LHC environment and it should provide further input to help the design of future detectors.

The core component of the DØ silicon detector is the Silicon Microstrip Tracker (SMT) [1] consisting of six barrels which are each $12 \mathrm{~cm}$ long and have seventy-two ladders arranged in four layers from Layer-1 (innermost) to Layer-4 (outermost). Each barrel is capped with a disk of wedge detectors ("F-disks") comprised of twelve wedges with trapezoidal shape. In the far forward (and backward) regions there are three additional F-disks and a large diameter "H-disk", to provide additional tracking at high pseudorapidity.

In order to compensate for the expected loss in performance due to radiation damage in the sensors in Layer-1, an additional layer was installed in 2006 for Run IIb of the Tevatron. This so-called Layer-0 [2] was built with LHC-based radiation-hard technology and is located inside the SMT. It consists of forty-eight silicon strip sensors mounted on six facets on a $1.68 \mathrm{~m}$ long, $1.6 \mathrm{~cm}$. radius carbon fiber support structure.

The CDF silicon detector consists of three different strucutures: The core component is the Silicon Vertex Detector (SVX) [3] which consist of six barrels each of them with sixty ladders in five layers (from inside, Layer-0 to Layer-4). The innermost layer is at a 2.5-3.0 cm distance from the beamline. The second component is the Intermediate Silicon Layers (ISL) [4] which are two layers of silicon strip sensors surrounding the SVX and which provide coverage to the forward region and also helps to link tracks from the SVX to the drift chamber of the CDF detector.

The last component of the CDF silicon detector is the Layer-00 [5] which is mounted on the Tevatron beampipe inside the SVX detector. The distance of the sensors to the beamline is $1.2-1.5 \mathrm{~cm}$. As in the case of Layer-0 of the $\mathrm{D} \emptyset$ detector, this layer was built using LHC-like technology providing higher radiation hardness and intended to improve tracking resolution and to help in maintaining the silicon-detector performance when the innermost layers of the SVX are compromised due to aging.

In the following sections we will describe the main studies performed within the two collaborations in order to evaluate the consequences of the radiation damage (aging) of the sensors and to monitor the evolution of the performance with time, with some special remarks on the expectations for the future of the silicon detectors in the plans for the Tevatron and the experiments. 


\section{Motivation for the studies on aging}

As mentioned above, the CDF and DØ silicon detectors are intended to facilitate the physics analysis of Tevatron collisions by providing precision tracking in a very challenging radiation environment. In addition to this, these detectors are being used for much longer time than was anticipated when originally designed. It should be remembered that these detectors were planned for the so-called Run IIa of the Tevatron, which was intended to last several years and in which the total collected luminosity would be of the order of $2-3 \mathrm{fb}^{-1}$. However, the cancellation of the replacements intended for the Run IIb upgrade forced the experiments to keep the current detectors much longer. In addition to this, the good performance of the Tevatron and the delays in the start-up of the LHC has led to unanticipated extensions of the time that the detectors need to be run, with the possible opportunity for Tevatron operations through more than $12 \mathrm{fb}^{-1}$ of accumulated luminosity.

These changes in the plans have re-enforced the fundamental need to monitor the status of the sensors in terms of the radiation damage and have prioritized the maintanance work required to keep the aging under control and keep the detectors fully functioning for as long as possible. On the other hand, the current plan provides also a strong motivation for performing the studies on radiation damage in a very unique situation, in which the sensors are strongly irradiated but during a long period of time, and during real operations of the detectors.

Although the effects caused by radiation are of several classes, for the purposes that the collaborations are interested in, the main concern is the bulk damage, especially displacement damage and defects in the crystal structure of the sensor which produce an increase of the shot noise, a change in the effective doping concentration and an increase of the charge carrier trapping. From the operational point of view, these effects translate into performance degradation due to the increase of noise from the sensors and a decrease of the collection efficiency of charge induced by charged particles crossing the sensors. In addition, the modification of the effective doping eventually causes the full depletion voltage to steadily increase and this sets a limit on the useful lifetime for which the sensor may be fully depleted, because of the maximum voltage that can be applied before sensor breakdown or because of the limited voltage from the power supply connected to the sensor. It should be noted that these limitations are due to the extended run of the Tevatron and the cancelled Run IIb upgrades, both directly affecting the performance of the detector and the strategy in operations.

As has been traditionally done, radiation damage is directly monitored by the study of the evolution of the bias current and of the full depletion voltage. The first method gives direct insight of the bulk condition and the effects from the absorbed radiation. The latter gives a more practical way of measuring the expected lifetime for operations with fully depleted sensors. Since neither of these two quantities provides quantitative information on the actual impact on physics performance, additional parallel variables are monitored and studied. Those are intended to provide direct information of the impact of aging on the detector performance and on the use of the silicon sensors as particle detectors. The most important of these quantities is the ratio of the average charge (MIP) over the noise of the sensor, which is called the signal-over-noise ratio $(\mathrm{S} / \mathrm{N})$, and the results of these studies are also described in detail below. Furthermore the study of other quantities, especially physics-related variables (as efficiencies, resolutions,...) as a function of the full depletion voltage has also become an useful tool, although still in development by the collaborations. 


\section{Studies of the leakage current}

The direct effect of the change in the bulk induced by the radiation is the increase of the leakage current in the biased sensors [6]. This current is the one measured through the PN junction when it is reversed biased and cannot be measured in the working sensors inside the CDF and DØ detectors, for which the bias current (i.e. the current measured though the bias lines when a bias voltage is applied) is the known quantity.

However, at the kind of doses at the Tevatron, it can be shown [7] that the observed increase in the bias current are dominated by the increase in the leakage current itself, and we obtain:

$$
\Delta I_{\text {bias }} \simeq \Delta I_{\text {leakage }}=\alpha \Delta \Phi
$$

where the second part of the equation is given by studies on radiation damage [6], $\Delta \Phi$ being the absorbed dose from a given starting point. The $\alpha$ parameter that relates both terms depends on the details of the sensor, but it is independent of the dose at first order, although there is some residual dependence for long periods of time due to annealing of the sensor.

At the Tevatron most (more than 99\%) of the radiation flux is produced by the actual physics collisions [7], so the integrated flux over time (fluence) is completely correlated to the accelerator integrated luminosity. Naturally the conversion function depends on the distance to the beam, and therefore the evolution of the bias currents is different for the different layers of sensors.

Measurements of the fluence have been obtained by the two collaborations from the variation in the bias current. As expected the innermost sensors are the most affected. This is quantified by analyzing the dependence of the fluence with the distance of the sensors to the beam $(R)$, which is parameterized as:

$$
\text { Fluence }=a \cdot R^{-\alpha}
$$

in which $a$ is a normalization constant independent of the distance, and the parameter $\alpha$ is fitted at $\mathrm{CDF}$ to a preferred value of $1.65 \pm 0.12$ with a $30 \%$ systematic uncertainty, dominated by the limited knowledge of the temperature and its profile along the sensors. The corresponding value from the $\mathrm{D} \emptyset$ study is $1.3 \pm 0.3$.

\section{Studies of the full depletion voltage}

The full depletion voltage is the bias voltage that is required to be applied to a sensor to get rid of all the free charges in the complete bulk. This condition is fundamental in order to reduce the noise of the sensor to a minimum level while enhancing the collection efficiency for charges generated during the ionization of the bulk induced by a crossing charge particle.

As radiation damage increases effects produced in the sensor lead to the type-inversion process in which the bulk changes the effective dope concentration and mutates from one type of doped bulk (e.g. $n$-type) to an effective opposite type ( $p$-like type). This change occurs in a continuous way as the sensor suffers more radiation and it is easily monitored with the full depletion voltage, which would show a decrease until the type inversion is complete. After that, the full depletion voltage continuosly increases. This behaviour [8] is expected for the internal sensors of the CDF 


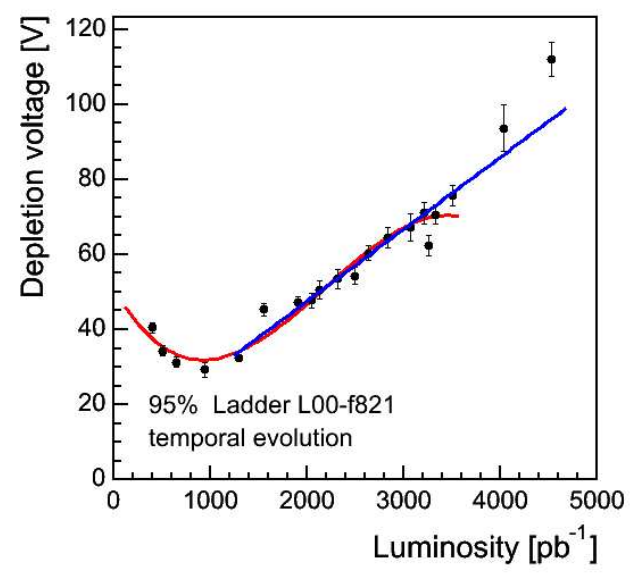

Figure 1: Evolution of the full depletion voltage of a sensor in CDF Layer-00 detector as a function of the integrated delivered luminosity. See text for details.

and $\mathrm{D} \emptyset$ silicon detectors and provides the information used to estimate the practical lifetime of the sensors. In order to perform these studies, it is required to measure the full depletion voltage at a given moment of time for each sensor. This is achieved by two methods: the signal-to-bias and the noise-to-bias scan techniques.

The signal-to-bias method consists of measuring the average charge collected for particles as a function of the bias voltage. As the voltage increases the efficiency to collect charge increases and thus the charge associated to the identified hits attached to reconstructed tracks becomes larger.

As we expect, the average charge for these identified hits increases as we increase the bias voltage until a maximum charge-collection efficiency is found. From this dependence we define the full depletion voltage as follows: The charge collection as a function of bias voltage is parameterized with a sigmoid function that is used to extract the full depletion voltage as the voltage value for which the corresponding charge is $95 \%$ the asymptotic value of the plateau.

This method is used by the two collaborations to measure the full depletion voltage in the innermost layers, and the value is monitored as the delivered luminosity increases.As shown in Fig. 1 the evolution of the full depletion voltage with integrated luminosity facilitates quantitative studies on the aging of specific sensors. The measured values at different times are fitted with a threedegree polynomial to extract the inversion point and with a straight line to obtain the behaviour after inversion and obtain expectations for the future. In the figure we observe the pattern that was expected for this kind of sensor: a decrease of the full depletion voltage until inversion is completed, and at the expected luminosity. From that moment we observe a clear linear increase of the full depletion voltage that would eventually reach the limit at which we could not fully deplete the sensor. As we describe below, linear fits are the functions that are used to extrapolate this evolution.

It should be noted that although the signal-to-bias method provide a very clean estimation of the full depletion voltage, it has two disadvantages: it does require beam-time and the collected data is not completely useful for physics, so it may affect data-collection efficiency However, the two collaborations take these calibration data when the detector has some problem that compromised the data for physics analysis. In addition to this, the signal-to-bias scan cannot be performed for 
the whole detector at the same time since it heavily relies on precise track reconstruction to identify good hit (and also for path-length corrections of the charge).

For these reasons, the collaborations also use an alternative method based on the measurement of the noise on the $n$-side of the sensors. This method only works for double-side sensors, in which the $n$ side is also read out. The idea is that as we are biasing the bulk, the full depletion area grows from the $p$-side (in which the PN junction occurs). This implies that the noise in the $n$-side is very insensitive to the full depletion taking place until the sensor is almost fully depleted. This description translates into the practical point, which is that when reading noise on the $n$-side as a function of the bias voltage a sudden decrease is observed at the full depletion voltage. This allows to extract the measurements for the whole detector in parallel. On the other hand the method does not work after inversion, since the previous picture is not valid any longer and the noise in the $n$-side and in the $p$-side is independent of the bias voltage.

The two collaborations differ on the way to actually extract the full depletion voltage: DØ uses the expectation of a "kink" in the noise as a function of the voltage for the double-sided doublemetal sensors. CDF fits a sigmoid function and uses the value for which the noise is $5 \%$ higher that the asymptotic value. This fitting procedure is also used by $\mathrm{D} \emptyset$ for the double-sided sensors.

Using this and the signal-to-bias method described above, $D \emptyset$ has produced the plots shown in Fig. 2, which shows the full depletion voltage, as measured with the two methods, as a function of the luminosity for a sensor in an inner layer and one for an outer layer. We observe that while the inner sensor has already inverted and show the expected rise of full depletion voltage as measured with the signal-to-bias scan, that is not the case for the outer sensor, which is still in the preinversion stage. The good ageeement between the two methods to extract the full depletion voltage is noteable. The difference is in the region close to and after the inversion point, where the noiseto-bias scan method is insensitive to the aging of the sensor and it is not useful to get a reliable measurement of the full depletion voltage.

\section{Signal-over-noise studies}

As mentioned above the monitoring of the aging of the sensors does not provide direct information on how the aging of the sensors is impacting the physics analyses for track reconstruction. For this reason, as part of the studies to monitor the aging, several additional and more physicsrelated quantities are computed.

On this topic the most common variable is the ratio of the most probable value of the charge collected for the hits in a particular sensor over the noise on the sensor. This signal-to-noise $(\mathrm{S} / \mathrm{N})$ ratio is estimated using real data for the signal and pedestal-calibration data for the noise, which provides access to a large amount of statistics without any additional effort from data accumulated during routine operation. Plot in Fig. 3 shows the result of the analysis of this variable for the sensors in the several layers of the CDF SVX detector.

The studies performed inside the two collaborations of the $\mathrm{S} / \mathrm{N}$ evolution with time shows that the performance of the sensors is going to be sufficient to be useful for analyses through the end of the run. At CDF the innermost layers display a clear decrease in $\mathrm{S} / \mathrm{N}$ but the extrapolation into the future yields a value that would stay above the qualitative reference for physics, although the trigger using the silicon detector (SVX) may be affected. Studies at D $\emptyset$ also yield similar results. 

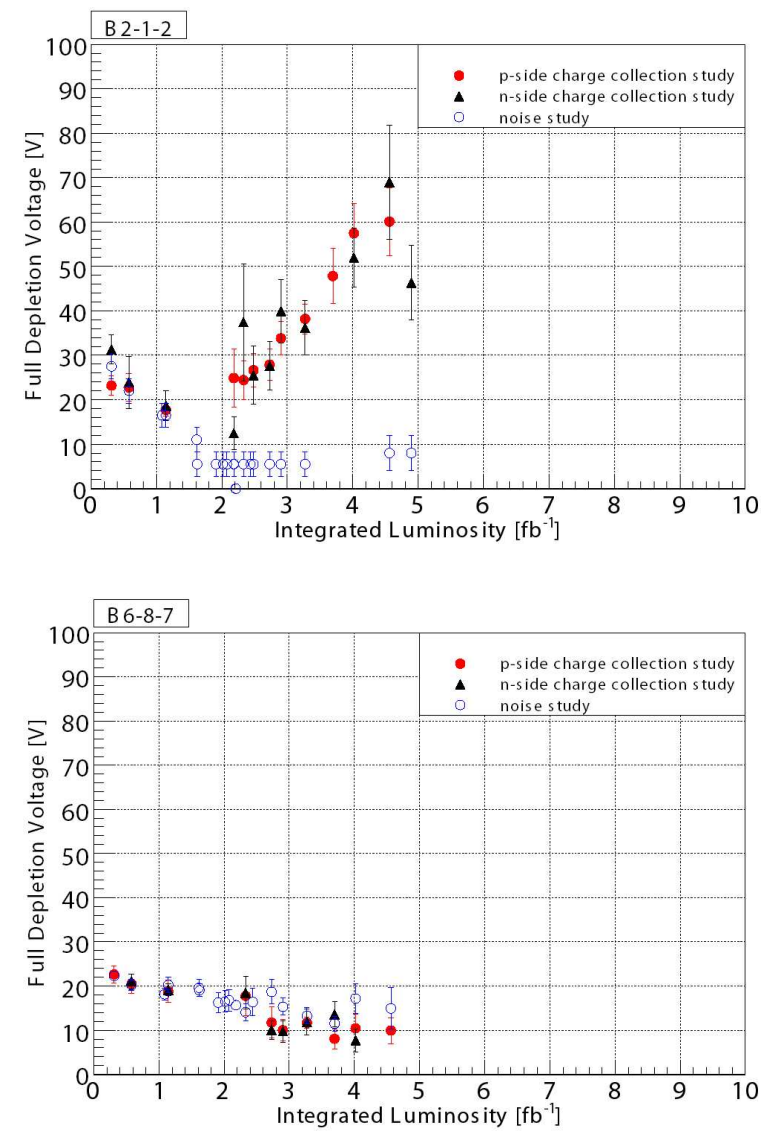

Figure 2: Measured values of the full depletion voltage as a function of the integrated delivered luminosity for an inner (left) and an outer sensor (right) at the DØ detector. Values extracted using the noise-to-bias (blue circles) and signal-to-bias (red dots and black triangles) methods are shown.

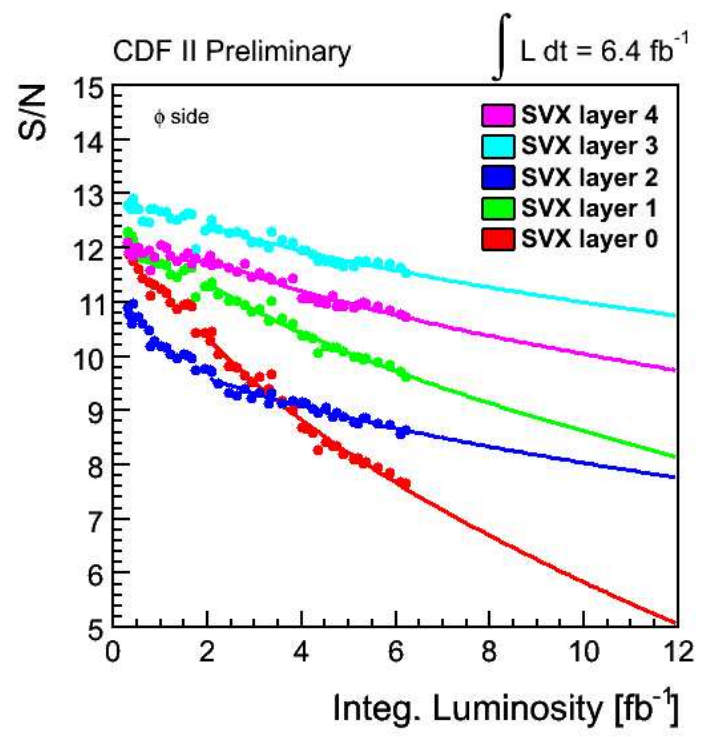

Figure 3: Evolution of the S/N of all the layers of the SVX detector of CDF as a function of the integrated luminosity. Extrapolated lines are included to show the expected values for this quantity in the future. 


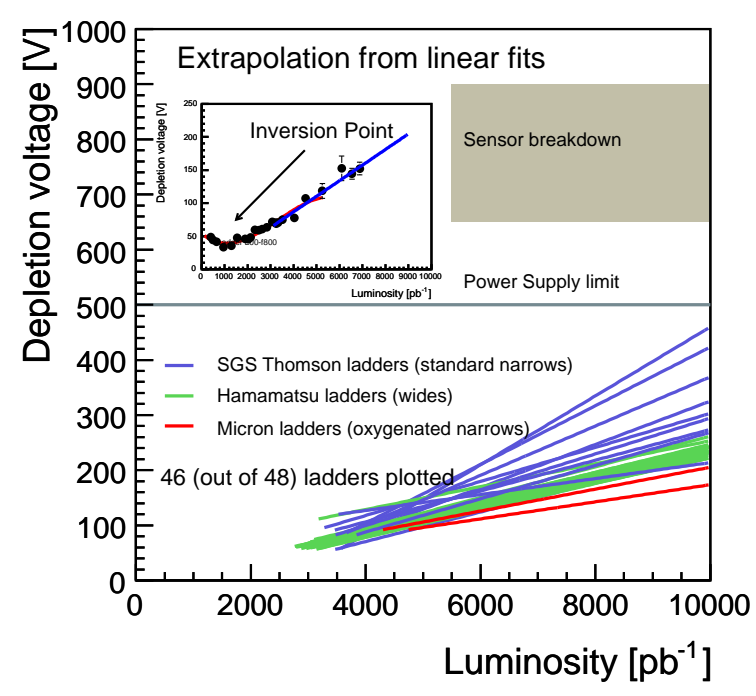

Figure 4: Expected results using linear extrapolations for the evolution of the full depletion voltage as a function of the integrated delivered luminosity at the Tevatron for the sensors in CDF Layer-00. Inset plot is equivalent to the one shown in Fig. 1.

\section{Expected lifetime of the detectors}

Although the S/N provides a reasonable reference about the future performance of the detectors, it assumes that conditions are not going to change. From the evolution of the full depletion voltage, is is clear that the main limitation for the innermost layer is coming from the bias voltage limit that at some point would prevent the sensors from being fully depleted. In those conditions it is expected that the noise level is increased and the collection charge not as efficient, and therefore the $\mathrm{S} / \mathrm{N}$ would degrade much faster than predicted by the extrapolations. Out of the studies on the full depletion voltage previously described it is possible to obtain linear predictions for the evolution of the full depletion voltage for the sensors, using the measured values. The results are shown in the plot of Fig. 4 for the CDF Layer-00 and in Fig. 5 for several layers of the DØ detector. We observe that the extrapolation show that the CDF sensors are going to be fine for most of the run, but required voltages may reach the power-supply limit beyond $10 \mathrm{fb}^{-1}$ of delivered luminosity. Similar conclusion may be drawn for SVX-L0, being the limit the sensor breakdown.

In the $D \emptyset$ case, the situation is different since the evolution of the full depletion voltage for the inner sensors of Layer- 1 suggest that around $8 \mathrm{fb}^{-1}$ the detectors will no longer be fully depleted. However, this was already anticipated and it was a main motivation for the Layer- 0 to be installed for Run IIb, as described before. It should be remarked that the sensors on this layer have not yet inverted and the expectations are that they will last for as long as needed, allowing to maintain the performance of the detector even when the inner sensors of Layer-1 can no longer be fully depleted.

Therefore these studies indicate that both silicon detectors will still provide valuable input to the reconstructed data for the rest of the run. Performance may be degraded relative to the beginning of the run, but expectations are that it will be high enough to continue serving as a fundamental part of the CDF and DØ track identification and reconstruction system. 
D $\varnothing$ Silicon Detector Radiation Aging Status as of July 2008

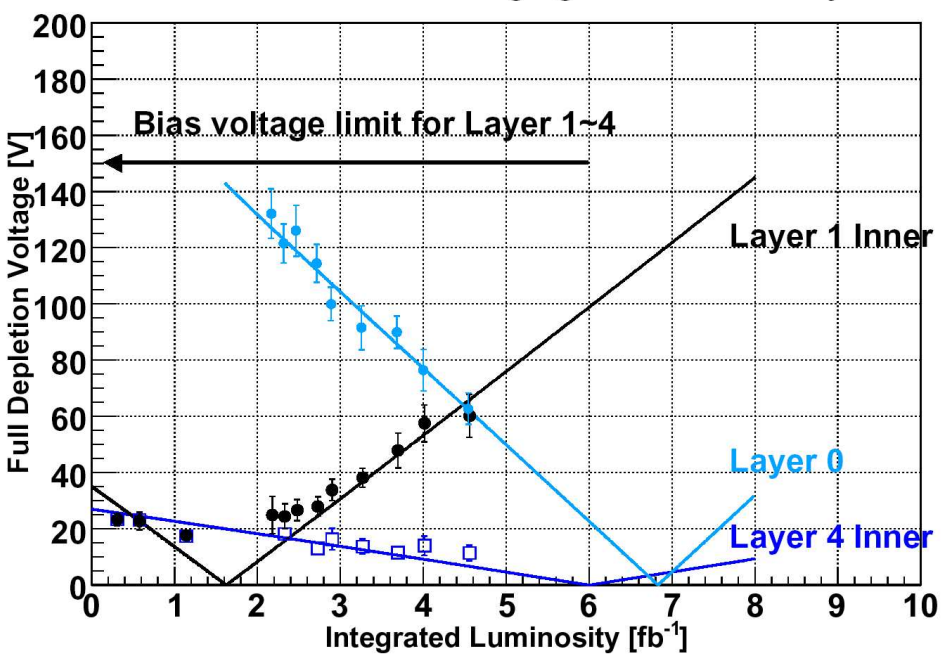

Figure 5: Expected results using linear extrapolations for the evolution of the full depletion voltage as a function of the integrated delivered luminosity at the Tevatron for some selected sensors in Layer-0, Layer-1 and Layer-4 of the $\mathrm{D} \varnothing$ detector.

\section{Conclusions}

The silicon detectors of the CDF and $\mathrm{D} \varnothing$ experiments at the Tevatron have been exposed to large radiation doses since 2001, much beyond what was originally planned. For this reason, they are continuosly monitored for radiation damage in order to keep them working as well as possible, due to the strong contraints coming out of the extension of the Tevatron running.

Several methods used for this monitoring are described in this report. The conclusion being that the tools are already in place and the sensors are withstanding the absorbed radiation at least as well as expected. Consequently both experiments should be able to make use of the precision tracking provided by the silicon detectors for as long as the Tevatron is running, even if extended to 2012 in order to achieve a total integrated delivered luminosity of $12-15 \mathrm{fb}^{-1}$, which is $5-6$ times larger that what was expected in the design for most of the currently running silicon sensors.

\section{References}

[1] V.M. Abazov et al. (The DØ Collaboration), Nucl. Instr. and Meth. A 565 (2006) 463.

[2] R. Angstadt et al, arXiv:0911.2522 submitted to Nucl. Instr. and Meth. A;

K. Hanagaki et al. (The DØ Collaboration), Nucl. Instr. and Meth. A 569 (2006) 8.

[3] A. Sill et al., Nucl. Inst. and Meth. A 447 (2000) 1.

[4] A. Affolder et al., Nucl. Inst. and Meth. A 453 (2000) 84.

[5] C.S.Hill et al., Nucl. Inst. and Meth. A 511 (2002) 118.

[6] M. Moll for the RD50 Collaboration, Nucl. Inst. and Meth. A 511 (2003) 97.

[7] R.J. Tesarek, Nucl. Instr. and Meth. A 514 (2003) 188-193.

[8] G. Lindström, M. Moll and E. Fretwurst, Nucl. Instr. and Meth. A 426 (1999) 1. 